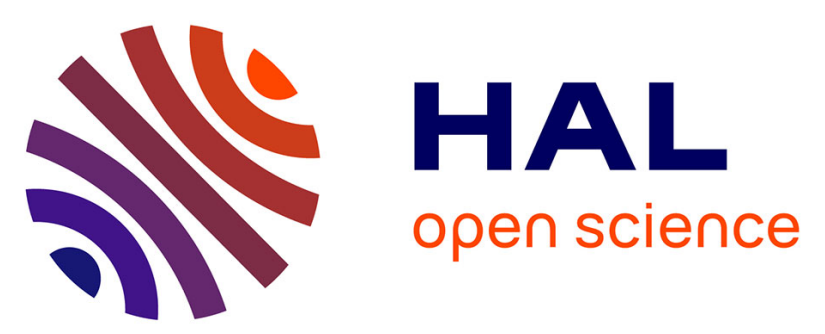

\title{
Effects of density dependent sex allocation on the dynamics of a simultaneous hermaphroditic population: Modelling and analysis
}

\author{
A. Kebir, S. Ben Miled, M.L. Hbid, R. Bravo de La Parra
}

\section{- To cite this version:}

A. Kebir, S. Ben Miled, M.L. Hbid, R. Bravo de La Parra. Effects of density dependent sex allocation on the dynamics of a simultaneous hermaphroditic population: Modelling and analysis. Journal of Theoretical Biology, 2010, 263 (4), pp.521. 10.1016/j.jtbi.2009.12.013 . hal-00578716

\author{
HAL Id: hal-00578716 \\ https://hal.science/hal-00578716
}

Submitted on 22 Mar 2011

HAL is a multi-disciplinary open access archive for the deposit and dissemination of scientific research documents, whether they are published or not. The documents may come from teaching and research institutions in France or abroad, or from public or private research centers.
L'archive ouverte pluridisciplinaire HAL, est destinée au dépôt et à la diffusion de documents scientifiques de niveau recherche, publiés ou non, émanant des établissements d'enseignement et de recherche français ou étrangers, des laboratoires publics ou privés. 


\section{Author's Accepted Manuscript}

Effects of density dependent sex allocation on the dynamics of a simultaneous hermaphroditic population: Modelling and analysis

A. Kebir, S. Ben Miled, M.L. Hbid, R. Bravo de la Parra

PII: S0022-5193(09)00580-3

DOI: doi:10.1016/j.jtbi.2009.12.013

Reference: YJTBI 5803

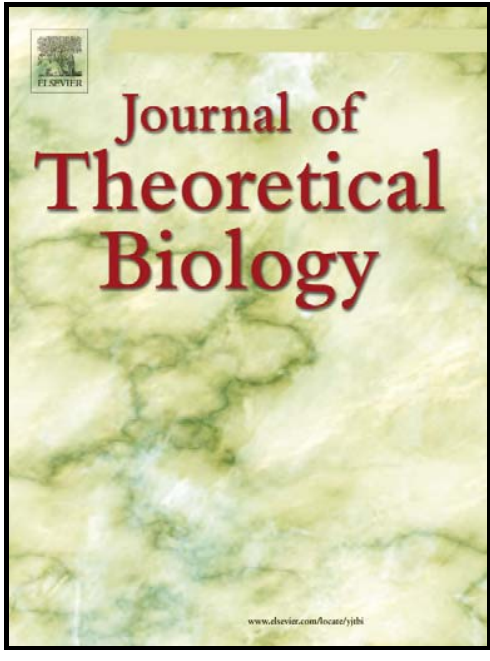

www.elsevier.com/locate/yjtb

To appear in: $\quad$ Journal of Theoretical Biology

Received date: 23 July 2009

Revised date: 5 December 2009

Accepted date: 14 December 2009

Cite this article as: A. Kebir, S. Ben Miled, M.L. Hbid and R. Bravo de la Parra, Effects of density dependent sex allocation on the dynamics of a simultaneous hermaphroditic population: Modelling and analysis, Journal of Theoretical Biology, doi:10.1016/j.jtbi.2009.12.013

This is a PDF file of an unedited manuscript that has been accepted for publication. As a service to our customers we are providing this early version of the manuscript. The manuscript will undergo copyediting, typesetting, and review of the resulting galley proof before it is published in its final citable form. Please note that during the production process errors may be discovered which could affect the content, and all legal disclaimers that apply to the journal pertain. 


\title{
Effects of Density Dependent Sex Allocation
} on the Dynamics of a Simultaneous

\section{Hermaphroditic Population: Modelling and}

\section{Analysis}

\begin{abstract}
A. Kebir ${ }^{\mathrm{a}, \mathrm{b}}$, S. Ben Miled ${ }^{\mathrm{a}, \mathrm{c}}$, M. L. Hbid ${ }^{\mathrm{b}}$
R. Bravo de la Parra d,*

${ }^{a}$ ENIT-LAMSIN, Tunis el Manar University, BP 37, 1002 Tunis Belvédère, Tunisia

${ }^{\mathrm{b}}$ LMDP, Cadi Ayyad University, BP 2390 Marrakech 4000, Morocco

${ }^{\mathrm{c}}$ Institut Pasteur de Tunis, 13, place Pasteur B.P. 74, 1002 Tunis Belvédère, Tunisia

${ }^{\mathrm{d}}$ Departamento de Matemáticas, Universidad de Alcalá, 28871 Alcalá de Henares, Spain \& UMI UMMISCO 209, IRD, 32 Av. Henri Varagnat, 93143 Bondy, France

Abstract

In this work we present a mathematical model describing the dynamics of a population where sex allocation remains flexible throughout adult life and so can be adjusted to current environmental conditions. We consider that the fractions of immature individuals acquiring male and female sexual roles are density dependent through non-linear functions of a weighted total population size. The main goal of this work is to understand the role of life-history parameters on the stabilization or destabilization of the population dynamics.
\end{abstract}

Preprint submitted to Elsevier

16 December 2009 
The model turns out to be a nonlinear discrete model which is analyzed by studying the existence of fixed points as well as their stability conditions in terms of model parameters. The existence of more complex asymptotic behaviours of system solutions is shown by means of numerical simulations.

Females have larger fertility rate than males. On the other hand, increasing population density favours immature individuals adopting the male role. A positive equilibrium of the system exists whenever fertility and survival rates of one of the sexual roles, if shared by all adults, allow population growing while the opposite happens with the other sexual role. In terms of the female inherent net reproductive number, $\eta_{F}$, it is shown that the positive equilibria is stable when $\eta_{F}$ is larger and closed to 1 while for larger values of $\eta_{F}$ a certain asymptotic assumption on the investment rate in the female function implies that the population density is permanent. Depending on the other parameters values, the asymptotic behaviour of solutions becomes more complex, even chaotic. In this setting the stabilization/destabilization effects of the abruptness rate in density dependence, of the survival rates and of the competition coefficients are analyzed.

Key words: Sex-allocation model, Sex-structured population dynamics, Density dependence, Stability analysis, Bifurcation and chaos

\section{$1 \quad 1 \quad$ Introduction}

2 In many organisms sex is a flexible affair, as it is for hermaphroditic and some

3 gonochoristic species with environmental sex determination (ESD), for which

\footnotetext{
* Corresponding author.

Email addresses: amira.kebir@lamsin.rnu.tn (A. Kebir), slimane@ipeit.rnu.tn (S. Ben Miled), hbid@ucam.ac.ma (M. L. Hbid), rafael.bravo@uah.es (R. Bravo de la Parra).
} 
4 environmental factors play a crucial role in their strategies of sexual resource

5 allocation. In fact, for these species the traits favored by one sex might be

6 costly to the other [Hosken and Stockley, 2005], what leads to a divergence

7 between male and female fitness and provides in the case of simultaneous

8 hermaphrodites a gender conflict [Angeloni, 2003, Anthes et al., 2006]. To

9 mediate this conflict, an evaluation of the current environmental conditions is

10 necessary [Charnov, 1982].

11 The Sex Allocation Theory [Ghiselin, 1969, Charnov, 1982, 1993] is a pow-

12 erful evolutionary theory that was developed to analyze the gender conflict

13 of such ESD species. This theory describes how the reproduction resources

14 are allocated between male and female components in order to maximize the

15 reproductive value, the fitness.

16 For outcrossing simultaneous hermaphrodites, the available literature [Charnov,

17 1982, Brauer et al., 2007, Schärer, 2009] shows that the mating group size in-

18 fluences sex allocation. Sex allocation theory for these species predicts a more

19 female-biased investment of reproductive resources when the mating group

20 size decreases [Fischer, 1981, Charnov, 1982, West et al., 2005, Anthes et al.,

21 2006, Shuker et al., 2007]. In relatively small groups individuals need to pro-

22 duce fewer sperms to be successful, thus leaving more resources for the female

23 role [Fischer, 1981, Charnov, 1982, Fischer, 1984]. Often the studies on local

24 mate competition for simultaneous hermaphrodites have focused on the evolu-

25 tionary adjustment of sex allocation to mating group size. As noted by Brauer

26 et al. [2007], there are, in fact, three different levels at which sex allocation can

27 be adjusted. First level, the allocation strategy can be the result of selection

28 and evolution, and thus be an adaptation to the average mating group size

29 over many generations. In the second case sex allocation is not or not strictly 
30 fixed genetically but it is set during ontogeny and therefore influenced by en-

31 vironmental conditions such as population density (developmental plasticity).

32 Finally, in the third level sex allocation is not fixed neither during evolution

33 nor ontogeny but remains flexible throughout adult life and can be adjusted to

34 current environmental conditions. In this work we represent sex allocation as

35 defined in the third case, therefore considering the density dependence of the

36 sex allocation as an evolutionary stable strategy. More precisely we suppose

37 that, at each reproductive cycle, the gender distribution of the population

38 changes with the fractions of immature individuals acquiring male and female

39 sexual roles being non-linear functions of a weighted total population size. We

40 have to notice that this approach has already been used for some (ESD) gono-

41 choristic species by many authors [Woodward and Murray, 1993] to explain

42 heavily biased sex-ratios, of order 10:1, in favor of females.

43 The main insights of this work have to do with how the general aspect of sexual

44 gender structures and the density dependence of sexual allocation may lead to

45 complex asymptotic behaviours. Mathematically speaking, we represent the

46 model by means of a structured discrete system with a density-dependent

47 form for immature sexual choice. Our aim is to understand the influence of

48 the intraspecific sexual competition on sex-ratio and population dynamics and

49 to generate information about stabilizing and destabilizing effects.

50 The paper is organized as follows. Firstly, we present the mathematical model

51 including the relevant biological features described previously. Then we pro-

52 ceed to the analytical study of the nonlinear discrete system by finding out,

53 whenever it is tractable, the existence of fixed points as well as their stability

54 conditions in terms of model parameters. We further discuss the asymptotic

55 behaviour of system solutions through numerical simulations where we observe 
56 a variety of complex chaos-like forms. Finally, we discuss the interpretation of

57 these results for the population dynamics of the referred species.

\section{$58 \quad 2 \quad$ The Model}

59 Let $J(t)$ denote the density of immature individuals, or juveniles, in the popu-

60 lation at times $t=0,1,2, \ldots$, where the unit of time is taken to be the duration

61 of a reproductive cycle. At the end of each reproductive cycle a fraction $s$ of

62 juveniles is assumed to keep being at this stage while a fraction $\pi$ come into

63 the mature stage, adulthood, and adopt either the male or the female func-

64 tions; the remaining fraction, $0<1-s-\pi \leq 1$, represents thus the juvenile

65 mortality rate per unit of time.

66 Juveniles when maturing to adulthood are faced to mating opportunities and

67 need to decide about which sexual role to adopt. They have the choice between

68 two genders: male or female. Let $M(t)$ and $F(t)$ denote the density of mature

69 individuals having adopted the male and the female functions respectively.

70 We assume that juveniles invest more in the most profitable sex role, thereby

71 getting a higher fertilization certainty [Anthes et al., 2006]. This investment

72 should then take into account the gender conflict between females, for which

73 multiple mating is advantageous, and males, for which avoiding female multi-

74 ple mating reduces the risk of competing with rival sperm. The sex allocation

75 is represented in the model through the investment rate in the female func-

76 tion, called $\Phi$, being then the investment rate in the male function $1-\Phi$. As

77 pointed out in the introduction we suppose sex allocation to be dependent on

78 population density through a weighted total population size: 


$$
W(t)=J(t)+\beta_{1} M(t)+\beta_{2} F(t),
$$

79 see [Cushing and Li, 1992] for a similar expression in a density dependent ju-

80 venile growth model, where positive parameters $\beta_{i}$ are competition coefficients

81 that measure the pressure effects of a male or female function individual on

82 the juveniles sexual role choice compare to that of a juvenile individual.

[Fig. 1 about here.]

84 In hermaphroditic species as well as in gonochoristic ones the fecundity of the

85 female function is limited to the amount of energy available for egg produc-

86 tion, while male function fecundity is limited to the available eggs (Bateman's

87 principle) [Bateman, 1948, Charnov, 1979]. Moreover, in relatively smaller

88 mating groups male individuals need to produce fewer sperm to be successful

89 in sperm competition, leaving more resources for the female function [Fischer,

90 1981, Charnov, 1982, Fischer, 1984, Brauer et al., 2007]. Applying these two

91 principles and assuming that the sizes of the population density and the cor-

92 responding average mating group are positively correlated, we consider the

93 investment rate in the female function $\Phi$ to be decreasing in terms of the

94 weighted total population size $W$, with all juveniles tending to join the female

95 (resp. male) class at maturation for very low (resp. high) population densities.

96 The survival rates of male and female adults are denoted $s_{1}$ and $s_{2}$ respectively.

97 Let $f_{2}$ denote the fertility rate of female adults. Also, assuming that during

98 the mating process an alternation of the sex role may be made by a small

99 number of males into the female role, Gamete Exchange [Anthes et al., 2006],

100 we denote $f_{1}$ the fertility rate of male adults, which should be much lower

101 than the one of females, i.e. $f_{1} \ll f_{2}$. 
102 Including all the elements defined so far we get the following nonlinear system

103 of difference equations:

$$
\left\{\begin{array}{l}
J(t+1)=s J(t)+f_{1} M(t)+f_{2} F(t) \\
M(t+1)=\pi(1-\Phi(W(t))) J(t)+s_{1} M(t) \\
F(t+1)=\pi \Phi(W(t)) J(t)+s_{2} F(t)
\end{array}\right.
$$

104 whose parameters are all positive and verify $s+\pi<1$ and $f_{1} \ll f_{2}$. Finally 105 we assume for function $\Phi$ :

$$
\Phi \in C^{1}\left(\mathbf{R}_{+},(0,1]\right), \Phi^{\prime}(x) \leq 0, \Phi(0)=1 \text { and } \lim _{x \rightarrow+\infty} \Phi(x)=0
$$

106 System (2) is an example of nonlinear autonomous matrix equation [Cushing, 107 1998]. Denoting $X=(J, M, F)$, system (2) can be written as,

$$
X(t+1)=P(X(t)) X(t)
$$

108 where,

$$
P(X(t))=P(W(t))=\left(\begin{array}{ccc}
s & f_{1} & f_{2} \\
\pi(1-\Phi(W(t))) & s_{1} & 0 \\
\pi \Phi(W(t)) & 0 & s_{2}
\end{array}\right)
$$

109 which is the sum, $P(X(t))=T(W(t))+F$, of the transition matrix, depending 
110 on the weighted total population size $W$, and the constant fertility matrix

$$
T(W)=\left(\begin{array}{ccc}
s & 0 & 0 \\
\pi(1-\Phi(W)) & s_{1} & 0 \\
\pi \Phi(W) & 0 & s_{2}
\end{array}\right) \quad \text { and } \quad F=\left(\begin{array}{ccc}
0 & f_{1} & f_{2} \\
0 & 0 & 0 \\
0 & 0 & 0
\end{array}\right) .
$$

\section{Model Analysis}

112 In this section we study the existence and stability of the equilibrium points

113 of system (2).

114 We use the net reproductive number, $\eta(W)$, of the projection matrix $P(W)$.

115 Matrix $P(W)$ is primitive for every positive $W$ and its strictly dominant 116 eigenvalue $r(W)$ verifies (Th 1.1.3 in [Cushing, 1998]): $r(W)>1$ if and only $117 \eta(W)>1, r(W)<1$ if and only $\eta(W)<1$ and thus $r(W)=1$ if and only $118 \eta(W)=1 . \eta(W)$ is the strictly dominant eigenvalue of matrix $F(I-T(W))^{-1}$ :

$$
F(I-T(W))^{-1}=\left(\begin{array}{ccc}
\frac{\pi f_{1}(1-\Phi(W))}{(1-s)\left(1-s_{1}\right)}+\frac{\pi f_{2} \Phi(W)}{(1-s)\left(1-s_{2}\right)} \frac{f_{1}}{1-s_{1}} \frac{f_{2}}{1-s_{2}} \\
0 & 0 & 0 \\
0 & 0 & 0
\end{array}\right)
$$

119 so 


$$
\eta(W)=\frac{\pi f_{1}}{(1-s)\left(1-s_{1}\right)}(1-\Phi(W))+\frac{\pi f_{2}}{(1-s)\left(1-s_{2}\right)} \Phi(W)
$$

120 This expression is simplified by denoting

$$
\eta_{M}=\frac{\pi f_{1}}{(1-s)\left(1-s_{1}\right)} \text { and } \eta_{F}=\frac{\pi f_{2}}{(1-s)\left(1-s_{2}\right)}
$$

121 that can be interpreted as the inherent net reproductive numbers (expected 122 number of offspring per newborn over the course of its lifetime) when only the 123 females reproduce and only the males reproduce respectively. We then have

$$
\eta(W)=\eta_{M}(1-\Phi(W))+\eta_{F} \Phi(W)
$$

124 Following the theory and methodology that can be found in [Cushing, 1998] 125 to perform a qualitative analysis of nonlinear autonomous matrix equation 126 like system (2), we study the existence and the stability of its extinction and 127 positive equilibria.

1283.1 Trivial equilibrium and extinction of the population.

129 The trivial, or extinction, equilibrium $\mathbf{0}=(0,0,0)$ of system (2) always ex130 ists. The Jacobian of system (2) at $\mathbf{0}$ is $P(0)$ and thus the facts of $\mathbf{0}$ being 131 asymptotically stable or unstable are directly expressed in terms of parameter $132 \eta_{F}=\eta(0)$.

133 Proposition 3.1 Let $\mathbf{0}=(0,0,0)$ be the trivial equilibrium of system (2). If

$134 \eta_{F}<1$, then $\mathbf{0}$ is asymptotically stable and if $\eta_{F}>1, \mathbf{0}$ is unstable. 
135 Proposition 3.1 yields that $\mathbf{0}$ loses its stability as $\eta_{F}$ increases through the

136 critical value 1 . We complete this bifurcation result in section 3.2 when con-

137 sidering the positive equilibria.

138 Under the hypothesis $s_{1} \leq s_{2}$, condition $\eta_{F}<1$ also implies that the trivial

139 equilibrium is globally stable, i.e., for any initial condition $(J(0), M(0), F(0)) \in$

$140 \mathbb{R}_{+}^{3}$ the corresponding solution $(J(t), M(t), F(t))$ tends to $\mathbf{0}$ as $t$ goes to infin-

141 ity.

142 Proposition 3.2 Let $s_{1} \leq s_{2}$. If $\eta_{F}<1$ then equilibrium 0 of system (2) is

143 globally asymptotically stable.

144 Proof: see appendix A.1.

1453.2 Positive Equilibria

146 In the next proposition we express in terms of $\eta_{M}$ and $\eta_{F}$ necessary and

147 sufficient conditions for system (2) to possess a positive equilibrium point

$148 X^{*}=\left(J^{*}, M^{*}, F^{*}\right)$. We also give conditions that ensures $X^{*}$ stability.

149 Proposition 3.3 System (2) has a positive equilibrium point $X^{*}=\left(J^{*}, M^{*}, F^{*}\right)$

150 if and only if either $\eta_{M}<1$ and $\eta_{F}>1$ or $\eta_{M}>1$ and $\eta_{F}<1$. In the first

151 case, $\eta_{M}<1, X^{*}$ is asymptotically stable for values of $\eta_{F}>1$ close enough to

1521 and, in the second case, $\eta_{M}>1, X^{*}$ is unstable for values of $\eta_{F}<1$ close

153 enough to 1.

154 Proof: see appendix A.2. 
155 The positive equilibrium, in both cases, can be explicitly calculated (see ap156 pendix A.2) and reads as follows:

$$
X^{*}=\left(J^{*}, \frac{\pi(1-\kappa)}{1-s_{1}} J^{*}, \frac{\pi \kappa}{1-s_{2}} J^{*}\right),
$$

157 where $J^{*}=\Phi^{-1}(\kappa) /\left(1+\beta_{1} \frac{\pi(1-\kappa)}{1-s_{1}}+\beta_{2} \frac{\pi \kappa}{1-s_{2}}\right)$ and $\kappa=\left(1-\eta_{M}\right) /\left(\eta_{F}-\eta_{M}\right)$.

158 Assuming $s_{1} \leq s_{2}$, it is also hold that $\eta_{M}<\eta_{F}$. We have already shown

159 that in this case if $\eta_{F}<1$ then the trivial equilibrium is globally stable while

160 for $\eta_{F}>1$ is unstable. Concerning the positive equilibrium $X^{*}$, the same 161 assumption reduces its existence conditions to $\eta_{M}<1<\eta_{F}$. In the next 162 proposition we prove that $s_{1} \leq s_{2}$ and $\eta_{M}>1$ imply unbounded solutions of 163 system (2).

164 Proposition 3.4 Let $s_{1} \leq s_{2}$ and $\eta_{M}>1$. If $(J(t), M(t), F(t))$ is the solution 165 of system (2) associated to the non-negative initial condition $(J(0), M(0), F(0)) \neq$ $166(0,0,0)$ then $\{(J(t), M(t), F(t)): t \in \mathbb{N}\}$ is unbounded.

167 Proof: see appendix A.3.

168 After that, we propose a sufficient condition for the permanence of the system 169 (2). We use the definition of permanence found in [Kon et al, 2004] which 170 ensures that the total population density neither explodes nor goes to zero.

171 To prove the permanence of system (2) we apply theorem 3 in [Kon et al, 2004];

172 for that we need system (2) to be dissipative, i.e., we need to find a compact 173 set $K \subset \mathbb{R}_{+}^{n}$ such that for all $X(0) \in \mathbb{R}_{+}^{n}$ there exists a $T=T(X(0))$ such 174 that $X(t) \in K$ for all $t \geq T$. In the next proposition we present a condition 175 on the investment rate in the female function, $\Phi$, that ensures system (2) 176 dissipativeness. 
177 Proposition 3.5 Let $\eta_{M}<1$. If $x \Phi(x)$ is bounded on $[0, \infty)$ then system (2)

178 is dissipative.

179 Proof: see appendix A.4.

180 Now, we state in the following proposition sufficient conditions for system (2)

181 permanence which is a direct consequence of theorem 3 in [Kon et al, 2004].

182 Proposition 3.6 Let $\eta_{M}<1$ and $\eta_{F}>1$. If $x \Phi(x)$ is bounded on $[0, \infty)$ then 183 system (2) is permanent.

184 In the next section we are presenting a set of simulations to complete the study

185 of those cases where the performed analysis does not help in characterizing the 186 asymptotic behaviour of solutions. These unknown cases correspond to the sit187 uation of existence of a unique positive equilibrium point, $X^{*}=\left(J^{*}, M^{*}, F^{*}\right)$, 188 for which it is not known whether it is stable or not. In terms of parameters $189 \eta_{M}$ and $\eta_{F}$, we are dealing with cases with a fixed $\eta_{M}<1$ and different values 190 of $\eta_{F}>1$. We should also choose a specific form for the allocation function $191 \Phi$ that verifies the condition imposed in proposition 3.6 so that system (2) is 192 permanent. This choice is in a sense robust because it can be proved, see chap193 ter 16 in [Hirsch and Smale, 1974], that if we take a perturbation $\left(C^{1}\right)$ of $\Phi$, 194 i.e., another allocation function $\tilde{\Phi}$ such that $|\Phi(x)-\tilde{\Phi}(x)|$ and $\left|\Phi^{\prime}(x)-\tilde{\Phi}^{\prime}(x)\right|$ 195 are small for every $x \in \mathbb{R}_{+}$, the existence of the positive equilibrium $X^{*}$ in the 196 case of $\Phi$, when it is hyperbolic, imply the existence of a close hyperbolic equi-

197 librium $\tilde{X}^{*}$ in the case of $\tilde{\Phi}$ which moreover shares the same stability features 198 with $X^{*}$. 
200 In this section we pursue the analysis of the asymptotic behaviour of solutions 201 of system (2) by means of numerical simulations.

202 We use particular forms of function $\Phi$, the investment rate in the female role.

203 In Getz [1996] it is analyzed the influence of abrupt density dependence on

204 the oscillations of a population by means of simple discrete models and us-

205 ing different density-dependent forms. Here we adapt the so-called generalized

206 Ricker function as presented in [Getz, 1996], $\Phi_{R_{\gamma}}(x)=e^{-x^{\gamma}}$, where the param-

207 eter $\gamma$ is called the "abruptness" parameter and controls how rapidly density 208 dependence sets in.

209 In the sequel we study how $\gamma$, the abruptness parameter, $s_{1}$ and $s_{2}$, the adult

210 survival rates, and $\beta_{1}$ and $\beta_{2}$, the competition coefficients, affect the stability of

211 the system. For this, we look for $\eta_{F}$, the female inherent reproductive number,

212 bifurcation values where the positive equilibrium point $X^{*}=\left(J^{*}, M^{*}, F^{*}\right)$

213 loses its stability as well as the values where the orbits that we calculate get

214 positive Largest Lyapunov Exponents (LLE).

2154.1 The Effect of the abruptness parameter $\gamma$ :

216 To illustrate the influence of the abruptness parameter $\gamma$ on the destabilization

217 of the positive equilibrium point $X^{*}$ we first calculate, figure A.2, the spectral

218 radius of its associated Jacobian matrix for different values of $\gamma \in\{1,1.5,2\}$

219 and $\eta_{F} \in[1,14]$ while keeping fixed the rest of parameters values. Let us recall

220 that Proposition 3.3 ensures the asymptotical stability of $X^{*}$ for $\eta_{F} \in(1,1+\varepsilon)$. 
221 It is shown in figure A.2 that this spectral radius is less than 1, i.e., $X^{*}$

222 asymptotically stable, for $\eta_{F}$ between 1 and a bifurcation value denoted $\eta_{F}^{*}$.

223 On the other hand, we see that $X^{*}$ is unstable for $\eta_{F}>\eta_{F}^{*}$.

[Fig. 2 about here.]

225 We notice that the larger the value of $\gamma$ the smaller the value of $\eta_{F}^{*}$, i.e, there 226 is a clear destabilizing effect produced by an increasing abruptness, the pa227 rameter that governs how rapidly an increase of the weighted total population 228 density is reflected on sex allocation going towards a more biased male distri229 bution.

230 To illustrate what happens once the positive equilibrium loses its stability we 231 use the results of a number of numerical simulations. We calculate orbits of 232 system (2), with initial conditions $X(0)=(J(0), M(0), F(0))=(20,15,10)$,

233 three different values of $\gamma \in\{1,1.5,2\}, \eta_{F} \in[0,80]$ and the rest of parameters 234 values being the same as those previously used. We also calculate the LLE of 235 each of these orbits.

236 In figure 3(a) it is shown for $\gamma=1$ the juvenile orbit diagram for values of $\eta_{F}$ 237 near biffurcation point, i.e., for each value of $\eta_{F}$ the points in its corresponding 238 vertical line approximate the long term behaviour of juveniles density $J(t)$, 239 thus they approximately represent the orbit attractor. It is noticed that for low 240 values of $\eta_{F}$ the asymptotic behaviour corresponds to the juvenile component $241 J^{*}$ of the positive equilibrium. The same is shown in figures $3(\mathrm{~b})$ and $3(\mathrm{c})$ for $242 \gamma=1.5$ and $\gamma=2$ respectively. 
244 In figure A.4, using $\eta_{F}$ as a parameter, the LLE of the orbits represented in

245 figure A.3 are plotted for the three different values of the abruptness parameter $246 \gamma \in\{1,1.5,2\}$. [Fig. 4 about here.]

248 To quantify the destabilizing effects of $\gamma$ we use, on the one hand, the low249 est value of $\eta_{F}$ from which positive Lyapunov exponents appear, henceforth 250 denoted $\eta_{F}^{\infty}$ (Fig. A.4), and, on the other hand, the amplitude of the orbit at251 tractors (Fig. A.3). We observe that increasing $\gamma$ entails decreasing $\eta_{F}^{\infty}$ while 252 there is an increase of the orbits attractors amplitude. We can then conclude 253 that an increase of parameter $\gamma$ promotes instability and chaotic-like oscilla254 tions in the population and that this kind of behaviour is reinforced as long 255 as the value of $\eta_{F}$ is increased. Larger sensibility to density dependence of 256 sex allocation together with larger female inherent rate reproductive number 257 implies more complex population dynamics.

258 4.2 The effect of the adult survival rates:

259 The survival rates $s_{1}$ and $s_{2}$ constitute important parameters of the model 260 since they characterize species iteroparity. To see their influence on the sta261 bility of the population dynamics we perform a numerical study analogous to 262 that already done for $\gamma$ in the previous section.

263 The first index we use to assess the stability of the population is $\eta_{F}^{*}$, the 264 bifurcation value of $\eta_{F}$ where the positive equilibrium $X^{*}$ of system (2) loses 265 its stability. We calculate $\eta_{F}^{*}$ for the different values of the survival rates $s_{1}$ 266 and $s_{2}$ in $[0.1,0.9]$, with $s_{1}<s_{2}$, and fixed values of the rest of parameters. In 
269 Understanding an increase of $\eta_{F}^{*}$ as a population stability rise, we notice the

270 clear stabilization effect of increasing the female survival rate. In fact, for any

271 fixed value of $s_{1}$ we see that the larger $s_{2}$ the bigger $\eta_{F}^{*}$. This is not the case

272 of $s_{1}$, as we can see that for certain values of $s_{2}$ the largest values of $\eta_{F}^{*}$ are

273 found for intermediate values for $s_{1}$ while the lowest values of $\eta_{F}^{*}$ are found

274 for either small or large values of $s_{1}$. This is certainly due to the asymmetric 275 roles of males and females.

276 Once the positive equilibrium $X^{*}$ loses its stability, our index to assess the 277 complexity of population dynamics are the values of $\eta_{F}^{\infty}$, that represent where 278 orbits start to possess positive Lyapunov exponents. We identify increasing $279 \eta_{F}^{\infty}$ with a gain in population stability. To obtain $\eta_{F}^{\infty}$ we calculate the orbits 280 of system (2), with the same conditions on $X(0)$ and $\eta_{F}$ as previously and 281 for the different values of $s_{1}$ and $s_{2}$ in $[0.1,0.9]$, and fixed values of the rest 282 of parameters. The 2D representation of $\eta_{F}^{\infty}$ in terms of $s_{1}$ and $s_{2}$ is shown 283 in figure A.6, where we notice that the complexity of population dynamics 284 increases as a result of low survival rates. High values of $s_{1}$ and $s_{2}$ imply 285 that very high values of $\eta_{F}$ are needed to get chaotic-like behaviour of system 286 orbits. This stabilization effect of the dynamical system can be assimilated to 287 iteroparity [Demetrius, 1971, Getz, 1996]: the repeated production of offspring 288 throughout the life cycle versus semelparity, where each individual reproduces 289 only once during its life. 
292 As the female role is more expensive in terms of reproductive energy, we 293 assume in this section that the female competition effect, represented by $\beta_{2}$, is 294 larger than the corresponding male effect, $\beta_{1}$, on juvenile gender choice, i.e. , $295 \beta_{2}>\beta_{1}$. The results in previous sections are not affected by this assumption.

296 We calculate $\eta_{F}^{*}$ for the different values of the competition coefficients $\beta_{1}$ and $297 \beta_{2}$ in $[0,50]$, with $\beta_{1}<\beta_{2}$, and fixed values of the rest of parameters as before. 298 In figure A.7, similarly to figure A.5, it is shown a $2 \mathrm{D}$ representation of $\eta_{F}^{*}$ in 299 terms of $\beta_{1}$ and $\beta_{2}$. We notice that the lowest values of $\eta_{F}^{*}$ are found for $\beta_{1}$ 300 either small or close to $\beta_{2}$.

[Fig. 7 about here.]

302 The results of the calculation of $\eta_{F}^{\infty}$, as done in previous sections, are shown 303 in figure A.8.

305 We note a clear destabilizing pattern, $\eta_{F}^{\infty}$ decreasing, directly related to the 306 increase of the male competition coefficient $\beta_{1}$. The fact that males and females are comparable in terms of reproductive energy entails a more complex population dynamics.

\section{Discussion and conclusion}

310 In this work we propose a model for the dynamics of an iteroparous out-

311 crossing simultaneous hermaphroditic population whose life cycle consists of 
312 a juvenile growth stage followed by a reproductive one with two different adult

313 sexual roles. At maturation period, the transition to the reproductive stage

314 depends on population density through a weighted total population size that

315 differentiates by gender the competition between juvenile and adult stages.

316 This approach suppose that sex allocation is not fixed during evolution but

317 remains flexible throughout adult life and can be adjusted to current environ-

318 mental conditions, this was already proved by many authors [Schärer, 2009],

319 our goal is to study the implication at a population dynamics level of this fact.

320 Apart from the parameters directly appearing in system (2) we have in321 troduced $\eta_{M}$ and $\eta_{F}$, the male and female inherent net reproductive num322 bers. We have proved that the system (2) has a positive equilibrium point $323 X^{*}=\left(J^{*}, M^{*}, F^{*}\right)$ if and only if either $\eta_{M}<1$ and $\eta_{F}>1$ or $\eta_{M}>1$

324 and $\eta_{F}<1$. Moreover, it exists a branch of non-extinction equilibrium pairs $325\left(\eta_{F}, X^{*}\right)$, which bifurcates from the point $\left(\eta_{F}, X^{*}\right)=(1,0)$ which results sta-

326 ble when $\eta_{M}<1$ and $\eta_{F} \gtrsim 1$ and unstable when $\eta_{M}>1$ and $\eta_{F} \lesssim 1$. Further 327 more, under condition $s_{1} \leq s_{2}$, we proved that if $\eta_{M}>1$ the population grows 328 unboundedly and if $\eta_{F}<1$ the population gets extinct. In the rest of the 329 discussion we assume $\eta_{M}<1$ and $\eta_{F}>1$, where we proved the permanence 330 of the system (2) provided that $x \Phi(x)$ be a bounded function for $x \in[0, \infty)$, 331 which is the case for the generalized Ricker function $\Phi_{R_{\gamma}}(x)=e^{-x^{\gamma}}$ used in 332 the numerical simulations of section 4 .

333 Parameter $\eta_{F}$ close to 1 represents, through $X^{*}$, low population density and 334 also female biased sex allocation due to density dependence effects. We proved 335 that $X^{*}$ is locally asymptotically stable whenever $\eta_{F}$ is bigger and close to 1 , 336 that is, a low population density implies a stabilization of the population with 
a sex allocation almost completely biased towards the female role. Density

339 Parameter $\eta_{M}$ close to 1 or high values of parameter $\eta_{F}$ represent high popu-

340 lation density at equilibrium $X^{*}$ what entails a decrease of the female invest-

341 ment rate. This situation corresponds to a loss of stability of the population

342 dynamics which, depending on other parameters values, may become chaotic.

343 In this context we studied the influence of density dependence through the

344 abruptness parameter $\gamma$. We found that the more severe the effects of den-

345 sity dependence are, i.e., the larger the parameter $\gamma$, the more complex the

346 population dynamics becomes. We have also studied the influence of survival

347 rates obtaining that the complexity of population dynamics increases if they

348 are low while high values of $s_{1}$ and specially of $s_{2}$ imply that very high values

349 of $\eta_{F}$ are needed to get chaotic-like behaviour of system orbits. An important

350 applied consequence of the last point is that selective mortality might have a

351 destabilization effect on exploited simultaneous hermaphroditic species. Con-

352 cerning competition parameters we also found a destabilizing effect when the

353 male coefficient $\beta_{1}$ is high, that is, when the reproductive energy consumed

354 by males and females is comparable.

355 We have shown, following the schema in Higgens [Higgins et al., 1997], that

356 the population dynamics shows a sensibility to changes in life history parame-

357 ters and in sex allocation patterns. Numerical simulations reveal the existence

358 of chaotic-like long-term behaviour of the population dynamics for certain

359 domains of parameters values. In this sense iteroparity and abruptness pa-

360 rameters have opposite effects. The latter is prone to imply chaotic behaviour

361 whereas the former promotes stability. Complex dynamics implies a strong

362 variability in the densities of male and female adults what induces also a 
364 of sex role changes per individual with the corresponding energy expenses. In

365 the case that this energy cannot be allocated, the concerned species has an evolutionary constraint in avoiding complex dynamics which should be counteracted by means of life history parameters such as $\eta_{F}, s, s_{1}$ and $s_{2}$.

368 It is implicit in model (2) that sex-allocation is regulated by two mechanisms 369 acting at two different time scales. The first one is an evolutionary mechanism

370 reflected in the existence of a sex-allocation function in terms of population 371 density and the second one has to do with the population dynamics derived

372 from this sex-allocation function. We proved, as the inherent rate reproductive 373 number increases, that this dynamics can be very complex to the point of ex-

374 hibiting chaotic-like attractors. However, we have to note that few experimen-

375 tal works exhibit these dynamical phenomena, perhaps because they suppose 376 that population density remains constant or absolute fitness values are mea377 sured as the breeding number instead of using the inherent net reproductive 378 rate, [Caswell, 2001], as we do. Integrating model (2) into an adaptative dy379 namics model for the traits $\beta_{1}$ and $\beta_{2}$, or $s_{1}$ and $s_{2}$, could help understanding 380 the relationships between the mentioned two times scales.

381 In general, sex allocation responses to environmental variability occurs at the 382 individual level. In our model, the study scale is at the population level and, 383 therefore, the response of the population to environmental changes should be 384 considered as the average of individual responses. To perform the study at the 385 individual level we could use a computational model like an IBM (individual 386 based model) which would allow subsequently a very interesting comparison 387 between both macroscopic and microscopic approaches. 
388 Finally, we have to note that the same sex allocation behaviour is observed

389 for some sequential hermaphroditic species. An analogous modelling approach

390 could be used in this case provided that the model integrates a new adult class

391 representing asexual individuals and the corresponding transitions from the

392 female and male classes to the asexual one after the reproductive period.

\section{A Appendix:}

394 A.1 Proof of proposition 3.2

$395 f_{1} \leq f_{2}$ and $s_{1} \leq s_{2}$ imply

$$
\left\{\begin{array}{l}
J(t+1)=s J(t)+f_{1} M(t)+f_{2} F(t) \leq s J(t)+f_{2}(M(t)+F(t)) \\
M(t+1)+F(t+1)=\pi J(t)+s_{1} M(t)+s_{2} F(t) \leq \pi J(t)+s_{2}(M(t)+F(t))
\end{array}\right.
$$

396 Considering now the linear system

$$
\left\{\begin{array}{l}
x(t+1)=s x(t)+f_{2} y(t) \\
y(t+1)=\pi x(t)+s_{2} y(t)
\end{array}\right.
$$

397 we find that $\eta_{F}<1$ implies that the strictly dominant eigenvalue of the 398 associated matrix is less than 1 and so for any initial condition $(x(0), y(0))$ 399 the corresponding solution verifies $\lim _{t \rightarrow \infty}(x(t), y(t))=(0,0)$.

400 On the other hand, for every $t \geq 0, J(t) \leq x(t)$ and $M(t)+F(t) \leq y(t)$ imply 
401 that $J(t+1) \leq x(t+1)$ and $M(t+1)+F(t+1) \leq y(t+1)$ :

402 Using (A.1) we have

$\left\{\begin{array}{l}J(t+1) \leq s J(t)+f_{2}(M(t)+F(t)) \leq s x(t)+f_{2} y(t)=x(t+1) \\ M(t+1)+F(t+1) \leq \pi J(t)+s_{2}(M(t)+F(t)) \leq \pi x(t)+s_{2} y(t)=y(t+1) .\end{array}\right.$

403 Now, for any initial condition $(J(0), M(0), F(0))$ of system $(2)$, setting $x(0)=$ $404 J(0)$ and $y(0)=M(0)+F(0)$ as initial conditions of system (A.2), the previous 405 inequalities imply that $J(t) \leq x(t)$ and $M(t)+F(t) \leq y(t)$ for every $t \geq 0$, 406 and so we obtain $\lim _{t \rightarrow \infty}(J(t), M(t), F(t))=(0,0,0)$.

\section{A.2 Proof of proposition 3.3 and equation (5)}

408 The condition for the existence of a positive equilibrium of system (2) (see 4091.2 .5 in [Cushing, 1998]) is that $\eta(W)=1$, i.e., $\eta_{M}(1-\Phi(W))+\eta_{F} \Phi(W)=1$ 410 or $\Phi(W)=\left(1-\eta_{M}\right) /\left(\eta_{F}-\eta_{M}\right)=\kappa$. Thus, there exists a positive equilibrium $411 X^{*}$ of system (2) if and only if $\kappa \in(0,1)$. In this case, $X^{*}=\left(J^{*}, M^{*}, F^{*}\right)$ is 412 unique and can be straightforwardly calculated as the eigenvector of matrix $413 P\left(\Phi^{-1}(\kappa)\right)$ associated to eingevalue 1 that verifies $\Phi\left(J^{*}+\beta_{1} M^{*}+\beta_{2} F^{*}\right)=\kappa$, 414 see (5).

415 The proof of proposition 3.3 is a direct consequence of the results in sec416 tion 1.2.2 of [Cushing, 1998]. Writing $P\left(\eta_{F}, X(t)\right)=T(W(t))+\eta_{F} \bar{F}$, where

$417 \bar{F}=\frac{1}{\eta_{F}} F$, and using $\eta_{F}$ as bifurcation parameter, theorem 1.2.4 ensures the 418 existence of a branch of positive equilibria bifurcating from the equilibrium 
419 pair $X=\overline{0}$ and $\eta_{F}=1$. The condition of existence of positive equilibria $420 \kappa \in(0,1)$ is equivalent to either $\eta_{M}<1$ and $\eta_{F}>1$ (bifurcation to the right) 421 or $\eta_{M}>1$ and $\eta_{F}<1$ (bifurcation to the left). Now applying theorem 1.2.6 of 422 [Cushing, 1998], as in our case $w^{T} B v=f_{2}(1-s) / \eta_{F}>0$, we obtain that the 423 bifurcation is stable if it is to the right and unstable if it is to the left, what 424 proves the proposition.

425 A.3 Proof of proposition 3.4

$426 f_{1} \leq f_{2}$ and $s_{1} \leq s_{2}$ imply

$$
\left\{\begin{array}{l}
J(t+1)=s J(t)+f_{1} M(t)+f_{2} F(t) \geq s J(t)+f_{1}(M(t)+F(t)) \\
M(t+1)+F(t+1)=\pi J(t)+s_{1} M(t)+s_{2} F(t) \geq \pi J(t)+s_{1}(M(t)+F(t))
\end{array}\right.
$$

427 Considering now the linear system

$$
\left\{\begin{array}{l}
x(t+1)=s x(t)+f_{1} y(t) \\
y(t+1)=\pi x(t)+s_{1} y(t)
\end{array}\right.
$$

428 Condition $\eta_{M}>1$ implies that the strictly dominant eigenvalue of the associ-

429 ated matrix is larger than 1 and so for any initial condition $(x(0), y(0)) \neq(0,0)$

430 the corresponding solution verifies $\lim _{t \rightarrow \infty}(x(t), y(t))=(+\infty,+\infty)$.

431 On the other hand, for every $t \geq 0, J(t) \geq x(t)$ and $M(t)+F(t) \geq y(t)$ imply

432 that $J(t+1) \geq x(t+1)$ and $M(t+1)+F(t+1) \geq y(t+1)$ : 


$$
J(t+1) \geq s J(t)+f_{1}(M(t)+F(t)) \geq s x(t)+f_{1} y(t)=x(t+1)
$$$$
M(t+1)+F(t+1) \geq \pi J(t)+s_{1}(M(t)+F(t)) \geq \pi x(t)+s_{1} y(t)=y(t+1) .
$$

434 Now, for any non-negative initial condition $(J(0), M(0), F(0)) \neq(0,0,0)$ of system (2), setting $x(0)=J(0)$ and $y(0)=M(0)+F(0)$ as initial conditions of system (A.4), the previous inequalities imply that $J(t) \geq x(t)$ and $M(t)+$ $F(t) \geq y(t)$, for every $t \geq 0$, and so that $\{(J(t), M(t), F(t)): t \in \mathbb{N}\}$ is 438 unbounded.

\section{A.4 Proof of proposition 3.5}

440 Let $K_{0}>0$ be such that $x \Phi(x)<K_{0}$ for all $x \geq 0$.

441 For every solution $X(t)=(J(t), M(t), F(t)) \in \mathbf{R}_{+}^{3}$ of system (2) we have

$$
F(t+1)=\pi \Phi(W(t)) J(t)+s_{2} F(t) \leq \pi \Phi(J(t)) J(t)+s_{2} F(t)<\pi K_{0}+s_{2} F(t)
$$

442 Since $0<s_{2}<1$, there exists $T_{1}=T_{1}(X(0))>0$ such that for $t \geq T_{1}(X(0))$

$$
F(t)<\frac{\pi K_{0}}{1-s_{2}}=K_{1}
$$

443 Now we have for all $t \geq T_{1}(X(0))$ that 


$$
\begin{gathered}
J(t+1)=s J(t)+f_{1} M(t)+f_{2} F(t)<s J(t)+f_{1} M(t)+f_{2} K_{1} \\
M(t+1)=\pi\left(1-\Phi(W(t)) J(t)+s_{1} M(t)<\pi J(t)+s_{1} M(t)\right.
\end{gathered}
$$

444 and thus

$$
\left(\begin{array}{c}
J(t+1) \\
M(t+1)
\end{array}\right)<\left(\begin{array}{cc}
s & f_{1} \\
\pi & s_{1}
\end{array}\right)\left(\begin{array}{c}
J(t) \\
M(t)
\end{array}\right)+\left(\begin{array}{c}
f_{2} K_{1} \\
0
\end{array}\right)
$$

445

$$
\left(\begin{array}{c}
J(t) \\
M(t)
\end{array}\right)<\left[\left(\begin{array}{ll}
1 & 0 \\
0 & 1
\end{array}\right)-\left(\begin{array}{cc}
s & f_{1} \\
\pi & s_{1}
\end{array}\right)\right]^{-1}\left(\begin{array}{c}
f_{2} K_{1} \\
0
\end{array}\right)=\frac{\eta_{F} K_{0}}{1-\eta_{M}}\left(\begin{array}{c}
1 \\
\frac{\pi}{1-s_{1}}
\end{array}\right)
$$

447 what completes the proof.

\section{References}

449 Angeloni L., 2003. Sexual selection in a simultaneous hermaphrodite with

450 hypodermic insemination: body size, allocation to sexual roles and paternity.

451 Animal Behaviour, 66(3):417-426.

452 Anthes N., Putz A., Michiels N. K., 2006. Sex role preferences, gender con- 
453 flict and sperm trading in simultaneous hermaphrodites: a new framework.

454 Animal Behaviour, 72(1):1-12.

455 Bateman A. J., 1948. Intra-sexual selection in drosophila. Heredity, 2:349-368.

456 Brauer V. S., Scharer L., Michiels N. K., 2007. Phenotypically flexible sex

457 allocation in a simultaneous hermaphrodite. Evolution, 61(1):216-222.

458 Caswell H., 2001. Matrix Population Models. Sinauer Associates Inc., Sunder459 land, Massachusetts.

460 Charnov E. L., 1979. Simultaneous hermaphroditism and sexual selection.

461 Proc Natl Acad Sci U S A, 76(5):2480-2484.

462 Charnov E. L., 1982. The theory of sex allocation. Monogr. Popul. Biol., Vol. 463 18, Princeton University Press.

464 Charnov E. L., 1993. Life History Invariants: Some Explorations of Symmetry 465 in Evolutionary Ecology (Broch). Oxford Series in Ecology 85 Evolution, 466 Oxford University Press.

467 Cushing J. M., 1998. An Introduction to Structured Population Dynamics. 468 CBMS-NSF Regional Conference Series in Applied Mathematics, Vol. 71, 469 SIAM, Philadelphia.

470 Cushing J. M., Li J., 1992. Intra-specific competition and density dependent 471 juvenile growth. Bulletin of mathematical biology, 54:503-519.

472 Demetrius L., 1971. Primitivity conditions for growth matrices. Mathematicals $473 \quad$ Biosciences, 12:53-58.

474 Fischer E. A., 1981. Sexual allocation in a simultaneously hermaphroditic 475 coral reef fish. Amer. Nat, 117:64-82.

476 Fischer E. A., 1984. Local mate competition and sex allocation in simultaneous 477 hermaphrodites. The American Naturalist, 124(4):590-596.

478 Getz W. M., 1996. A hypothesis regarding the abruptness of density depen- 
480 Ghiselin M., 1969. The evolution of hermaphroditism among animals. The 481 Quarterly Review. of Biology, 44:189-208.

482 Higgins K., Hastings A., Botsford L. W., 1997. Density dependence and age 483 structure: Nonlinear dynamics and population behavior. The American $484 \quad$ Naturalist, 149(2):247-269.

485 Hirsch M. W., Smale S., 1974, Academic Press, Inc.: Differential Equations, 486 Dynamical Systems, and Linear Algebra. San Diego.

487 Hosken D. J., Stockley P. 2005. Sexual conflict. Current Biology, 15(14): $488 \quad 535-536$.

489 Kon R., Saito Y., Takeuchi Y. 2004, Permanence of single-species stage490 structured models. Mathematical Biology, 48(5):515-528.

491 Schärer L. 2009. Tests of Sex Allocation Theory in Simultaneously 492 Hermaphroditic Animals. Evolution, 63(6):1377-1405.

493 Shuker D. M., Reece S. E., Lee A., Graham A., Duncan A. B., West S. A., 494 2007. Information use in space and time: sex allocation behaviour in the 495 parasitoid wasp nasonia vitripennis. Animal Behaviour, 73(6):971-977.

496 Stewart G.W., Sun Ji-guang, 1990, Academic Press, Inc.: Matrix Perturbation 497 Theory. Boston.

498 West S. A., Shuker D. M., Sheldon B. C., 2005. Sex-ratio adjustment when 499 relatives interact: A test of constraints on adaptation. Evolution, 59(6): $500 \quad 1211-1228$.

501 Woodward D. E., Murray J. D., 1993. On the Effect of Temperature502 Dependent Sex Determination on Sex Ratio and Survivorship in Crocodil503 ians. Proceedings of the Royal Society B: Biological Sciences, 252:149-155. 
A.2 The spectral radius of the Jacobian matrix associated to the equilibrium point $X^{*}=\left(J^{*}, M^{*}, F^{*}\right)$ of system (??) is shown for three different values of $\gamma \in\{1,1.5,2\}$, as $\eta_{F}$ increases from 1 to 14 , with $s=0.05, s_{1}=0.25, s_{2}=0.4, \pi=0.6, f_{1}=0.01$, $f_{2}=\eta_{F}(1-s)\left(1-s_{2}\right) / \pi, \beta_{1}=1, \beta_{2}=1.5$ and $\Phi(x)=e^{-x^{\gamma}}$.

A.3 Using the female inherent reproductive number $\eta_{F}$ as a parameter, orbit diagrams for the juvenile density $J$ are shown for system (??) with parameter values $s=0.05, s_{1}=0.25, s_{2}=0.4, \pi=0.6, f_{1}=0.01$, $f_{2}=\eta_{F}(1-s)\left(1-s_{2}\right) / \pi, \beta_{1}=1$ and $\beta_{2}=1.5$, initial conditions $X(0)=(J(0), M(0), F(0))=(20,15,10)$, and $\Phi(x)=e^{-x_{\gamma}^{\gamma}}:(\mathrm{a})$ $\gamma=1$, (b) $\gamma=1.5$ and (c) $\gamma=2$ (note the different vertical scales).

A.4 The largest Lyapunov exponents (LLE) of the orbit of system (??) is shown for three different values of $\gamma \in\{1,1.5,2\}$, as $\eta_{F}$ increases from 0 to 22 , with parameters values and initial conditions as in Fig. ??. $\eta_{F}^{\infty}$ represents the largest value of $\eta_{F}$ such that the LLE of the orbit is negative for $\eta_{F} \leq \eta_{F}^{\infty}$.

A.5 The value $\eta_{F}^{*}$, value of $\eta_{F}$ for which the positive equilibrium point $X^{*}=\left(J^{*}, M^{*}, F^{*}\right)$ of system (??) loses its stability, is shown for $s_{1} \in[0.1,0.9]$ and $s_{2} \in\left[s_{1}, 0.9\right]$, with $s=0.05$, $\pi=0.6, f_{1}=0.01, \beta_{1}=1, \beta_{2}=1.5$ and $\Phi(x)=e^{-x}$.

A.6 The value $\eta_{F}^{\infty}$, smallest value of $\eta_{F}$ starting from which the corresponding orbit has a positive LLE, is shown for $s_{1} \in[0.1,0.9], s_{2} \in\left[s_{1}, 0.9\right]$, with $s=0.05, \pi=0.6, f_{1}=0.01$, $\beta_{1}=1, \beta_{2}=1.5$ and $\Phi(x)=e^{-x}$.

A.7 The value $\eta_{F}^{*}$, value of $\eta_{F}$ for which the positive equilibrium point $X^{*}=\left(J^{*}, M^{*}, F^{*}\right)$ of system (??) loses its stability, is shown for $\beta_{1} \in[0,50]$ and $\beta_{2} \in\left[\beta_{1}, 50\right]$, with $s=0.05$, $s_{1}=0.25, s_{2}=0.4, \pi=0.6, f_{1}=0.01$ and $\Phi(x)=e^{-x}$.

A.8 The value $\eta_{F}^{\infty}$, smallest value of $\eta_{F}$ starting from which $\beta_{1} \in[0,50]$ and $\beta_{2} \in\left[\beta_{1}, 50\right]$, with $s=0.05, s_{1}=0.25, s_{2}=0.4$, $\pi=0.6, f_{1}=0.01$ and $\Phi(x)=e^{-x}$. 
Adult

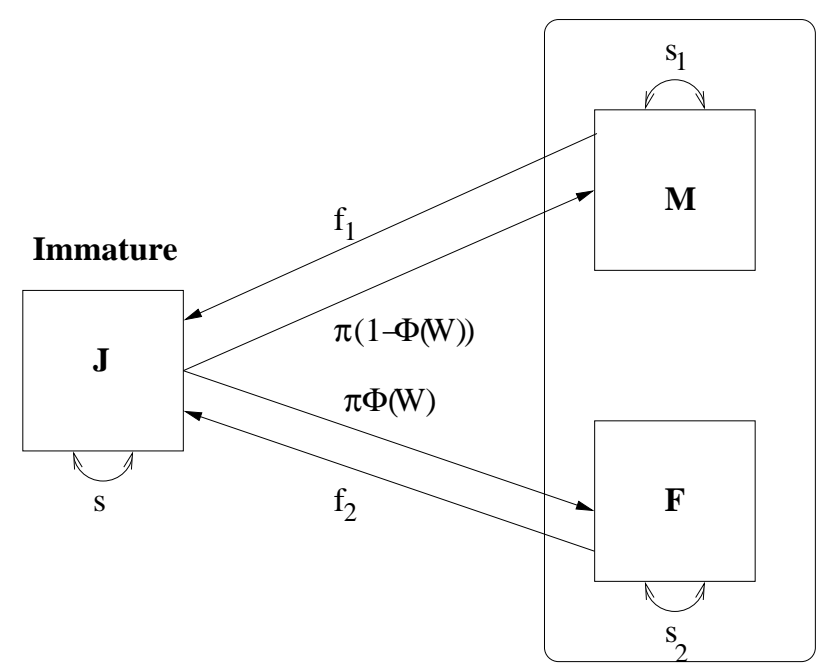

Fig. A.1. Conceptual model. 


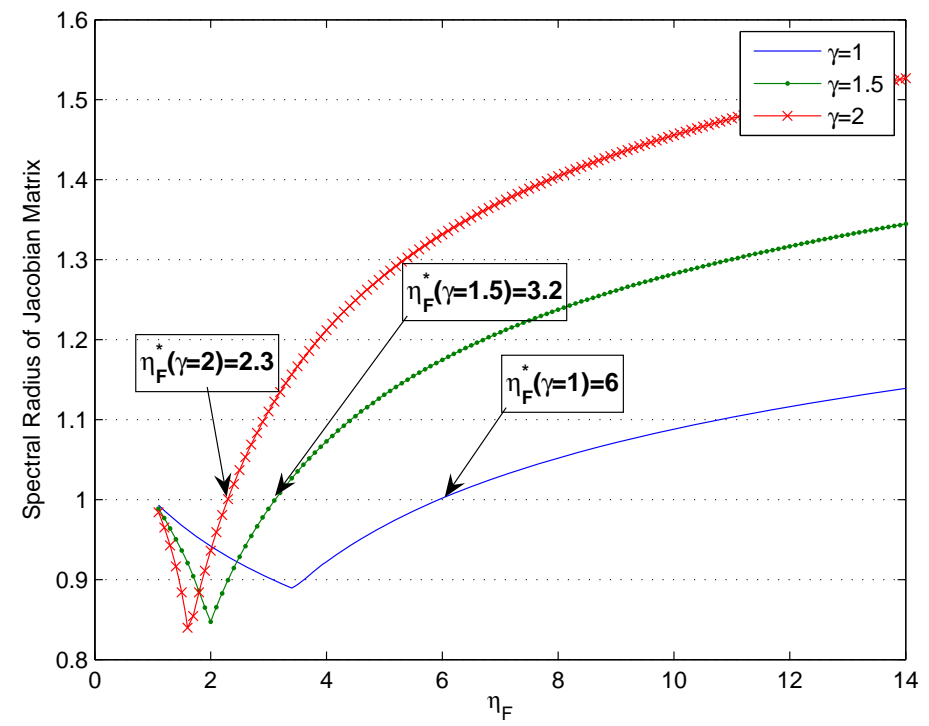

Fig. A.2. The spectral radius of the Jacobian matrix associated to the equilibrium point $X^{*}=\left(J^{*}, M^{*}, F^{*}\right)$ of system (2) is shown for three different values of $\gamma \in\{1,1.5,2\}$, as $\eta_{F}$ increases from 1 to 14 , with $s=0.05, s_{1}=0.25, s_{2}=0.4$, $\pi=0.6, f_{1}=0.01, f_{2}=\eta_{F}(1-s)\left(1-s_{2}\right) / \pi, \beta_{1}=1, \beta_{2}=1.5$ and $\Phi(x)=e^{-x^{\gamma}} \cdot \eta_{F}^{*}$ represents the bifurcation value where $X^{*}$ loses its stability. 


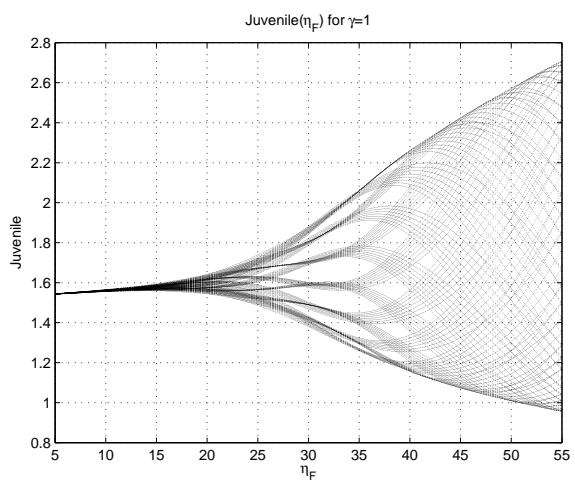

(a) $\gamma=1$

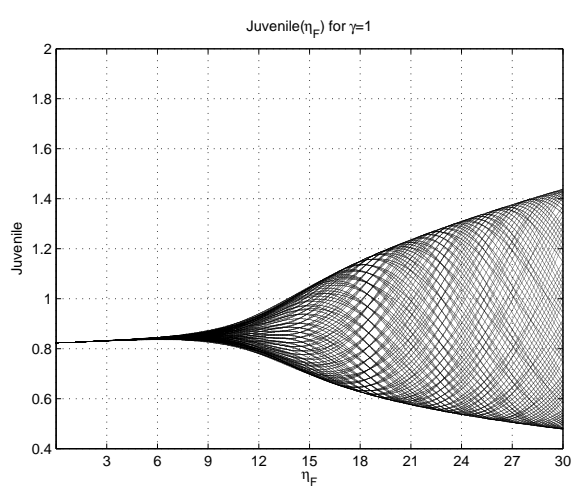

(b) $\gamma=1.5$

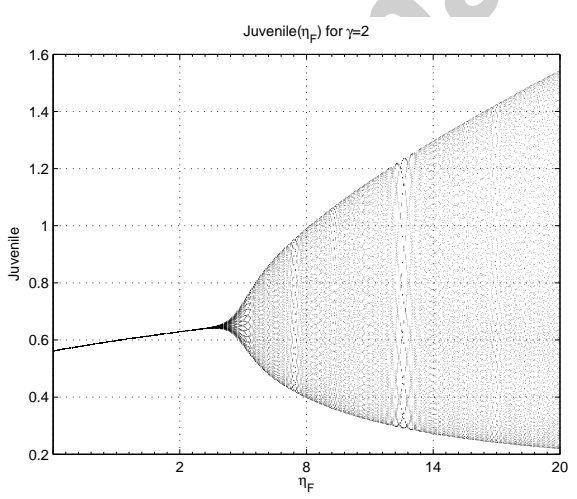

(c) $\gamma=2$

Fig. A.3. Using the female inherent reproductive number $\eta_{F}$ as a parameter, orbit diagrams for the juvenile density $J$ are shown for system (2) with parameter values $s=0.05, s_{1}=0.25, s_{2}=0.4, \pi=0.6, f_{1}=0.01, f_{2}=\eta_{F}(1-s)\left(1-s_{2}\right) / \pi$, $\beta_{1}=1$ and $\beta_{2}=1.5$, initial conditions $X(0)=(J(0), M(0), F(0))=(20,15,10)$, and $\Phi(x)=e^{-x^{\gamma}}$ : (a) $\gamma=1$, (b) $\gamma=1.5$ and (c) $\gamma=2$ (note the different vertical scales). 


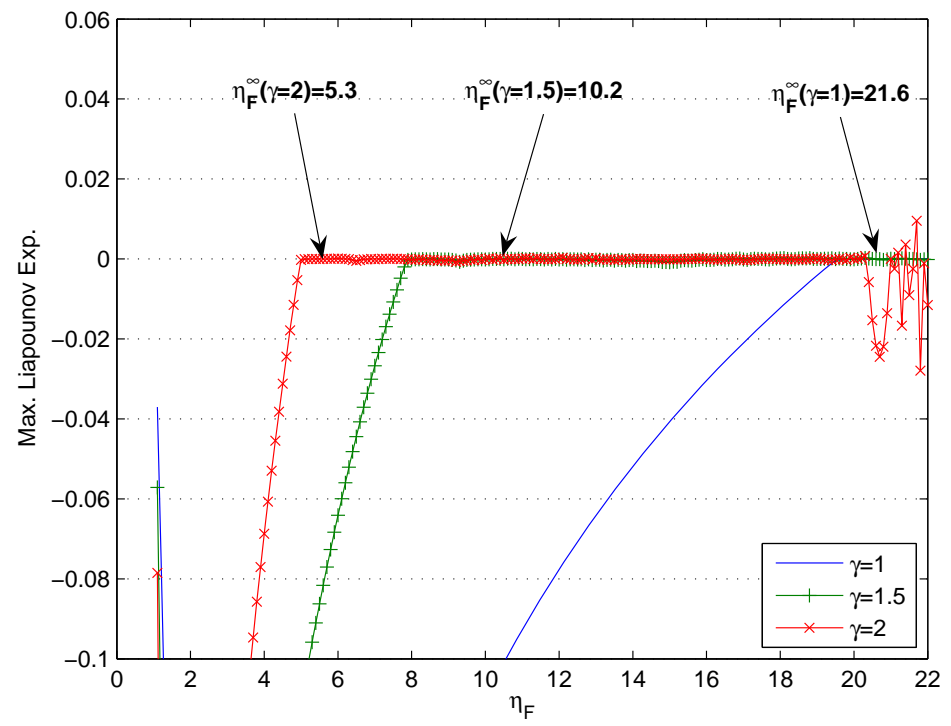

Fig. A.4. The largest Lyapunov exponents (LLE) of the orbit of system (2) is shown for three different values of $\gamma \in\{1,1.5,2\}$, as $\eta_{F}$ increases from 0 to 22 , with parameters values and initial conditions as in Fig. A.3. $\eta_{F}^{\infty}$ represents the largest value of $\eta_{F}$ such that the LLE of the orbit is negative for $\eta_{F} \leq \eta_{F}^{\infty}$. 


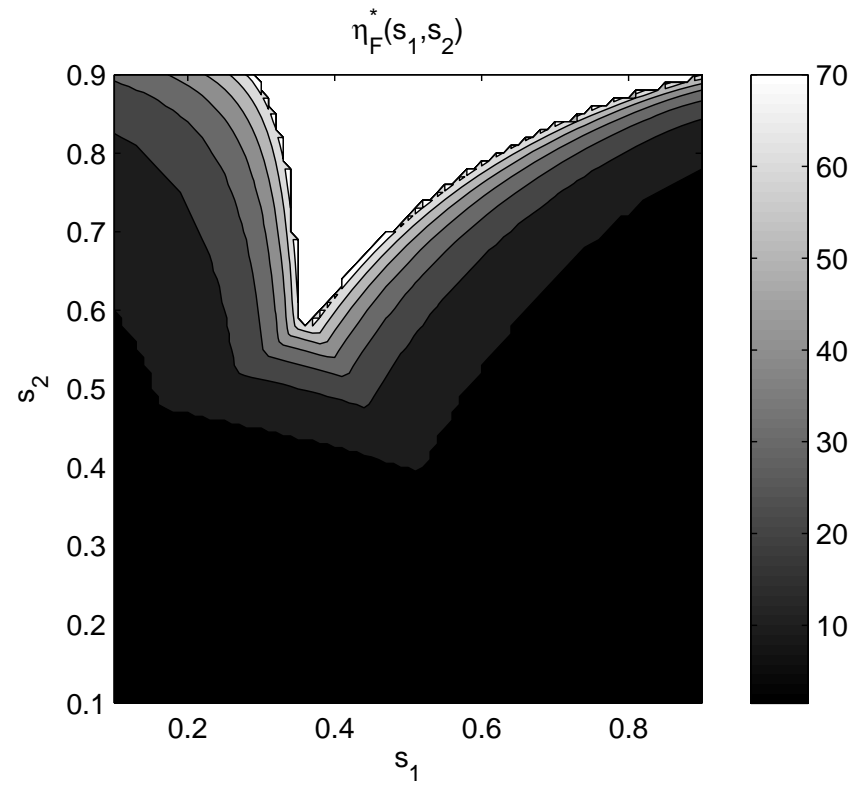

Fig. A.5. The value $\eta_{F}^{*}$, value of $\eta_{F}$ for which the positive equilibrium point $X^{*}=\left(J^{*}, M^{*}, F^{*}\right)$ of system (2) loses its stability, is shown for $s_{1} \in[0.1,0.9]$ and $s_{2} \in\left[s_{1}, 0.9\right]$, with $s=0.05, \pi=0.6, f_{1}=0.01, \beta_{1}=1, \beta_{2}=1.5$ and $\Phi(x)=e^{-x}$. 


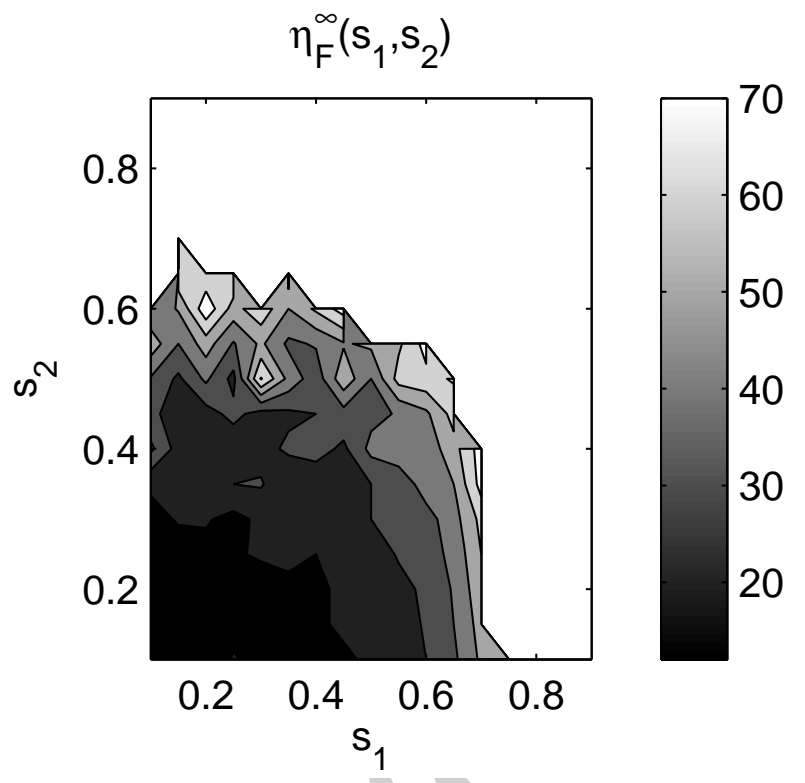

Fig. A.6. The value $\eta_{F}^{\infty}$, smallest value of $\eta_{F}$ starting from which the corresponding orbit has a positive LLE, is shown for $s_{1} \in[0.1,0.9], s_{2} \in\left[s_{1}, 0.9\right]$, with $s=0.05$, $\pi=0.6, f_{1}=0.01, \beta_{1}=1, \beta_{2}=1.5$ and $\Phi(x)=e^{-x}$. 


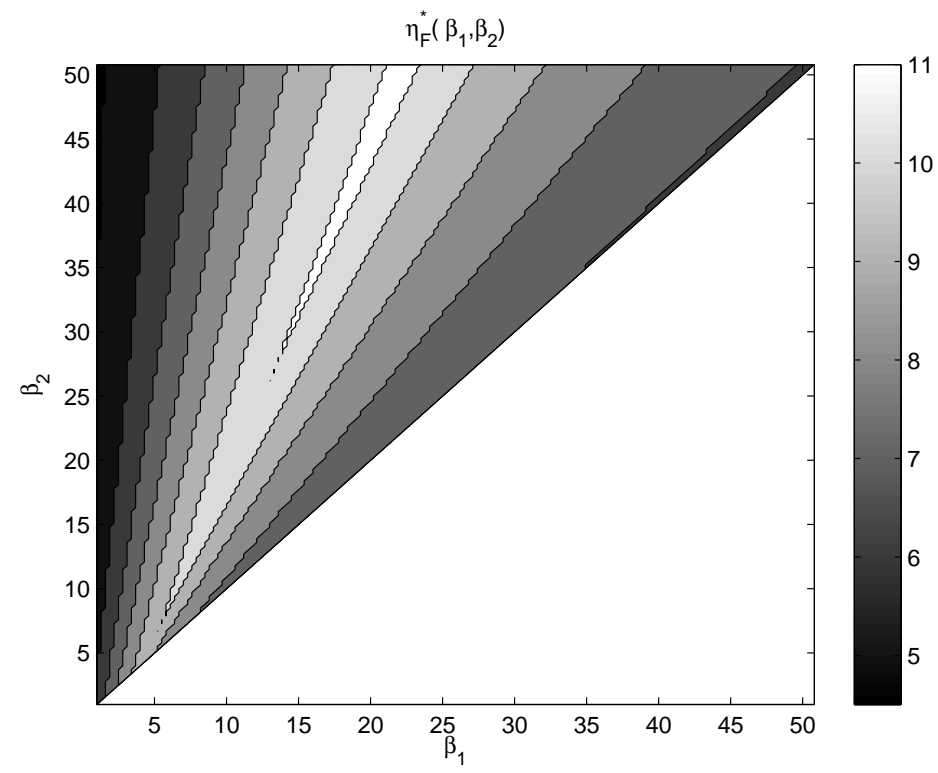

Fig. A.7. The value $\eta_{F}^{*}$, value of $\eta_{F}$ for which the positive equilibrium point $X^{*}=\left(J^{*}, M^{*}, F^{*}\right)$ of system (2) loses its stability, is shown for $\beta_{1} \in[0,50]$ and $\beta_{2} \in\left[\beta_{1}, 50\right]$, with $s=0.05, s_{1}=0.25, s_{2}=0.4, \pi=0.6, f_{1}=0.01$ and $\Phi(x)=e^{-x}$. 


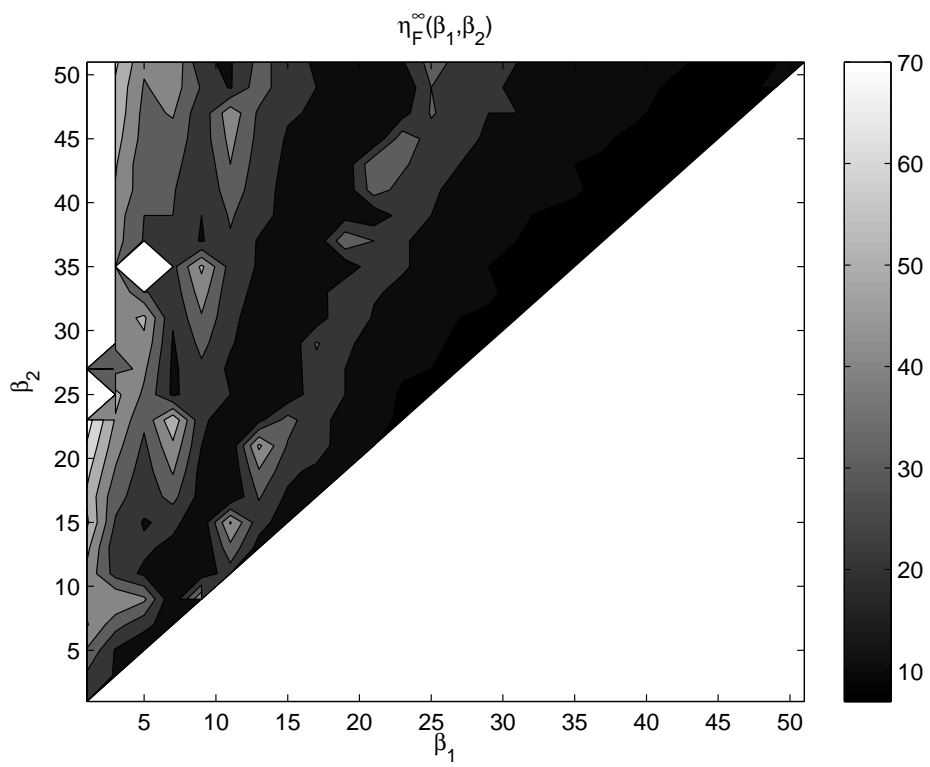

Fig. A.8. The value $\eta_{F}^{\infty}$, smallest value of $\eta_{F}$ starting from which the corresponding orbit has a positive LLE, is shown for $\beta_{1} \in[0,50]$ and $\beta_{2} \in\left[\beta_{1}, 50\right]$, with $s=0.05$, $s_{1}=0.25, s_{2}=0.4, \pi=0.6, f_{1}=0.01$ and $\Phi(x)=e^{-x}$. 